\title{
Defect strength and strain glass state in ferroelastic systems
}

\author{
Dong Wang ${ }^{1,2}$, Duchao Lv ${ }^{1,2}$, Yipeng $\mathrm{Gao}^{2}$, Yu Wang $^{l^{*}}$, Xiaobing Ren ${ }^{1,3}$ and Yunzhi Wang ${ }^{1,2}$
}

1. Frontier Institute of Science and Technology, MOE Key Laboratory for

NonequilibriumSynthesis and Modulation of Condensed Matter and State Key Laboratory for

Mechanical Behavior of Materials, Xi'an Jiaotong University, Xi'an 710049, China

2. Department of Materials Science and Engineering, The Ohio State University, 2041 College

Road, Columbus, OH 43210, USA

3. Ferroic Physics Group, National Institute for Materials Science, Tsukuba, 305-0047, Ibaraki,

Japan

\begin{abstract}
It has been shown both experimentally and by computer simulations that stress-carrying defects can change a normal sharp first-order martensitic transformation (MT) to a continuous strain glass transition in ferroelastic systems and offer unique properties. However, strain glass state has been found only in limited ferroelastic systems with relatively small transformatoin strains such as the trigonalphase $(1 \%)$ in NiTi-based alloys and the orthorhombicphase $(2 \%)$ in TiNb-based alloys by means of impurity doping. We show in this paper that there existsa critical value of defect strength relative to the strength of MTs for creating a strain glass state. Using a typical strain glass system, $\mathrm{Ti}_{48.5} \mathrm{Ni}_{51.5}$ with symmetrical anti-site defects,as an example, we show that the equivalent Von Mises strain caused by anti-site defect with randomly distribution (with 3\% concentration) is $7 / 8.9$ times of that created by the stress free transformation strain of Martensitic phase in order to produce an R/B19 strain glass state respectively. Themaximum interaction energy between the anti-site defects and the martensitic variant isestimated to be 16 times larger than that between different martensitic variants at this defect concentration. This finding may shed light on developing new strain glasses of much larger transformation strains for broader applications through defect engineering.
\end{abstract}

Keywords:Martensitictransformation, defect engineering, strain glass, phase field

method

* Corresponding author:

yuwang@mail.xjtu.edu.cn 


\section{Introduction}

Martensitic transformation (MT) is a diffusionless and shear-dominant solid-state structural phase transformation found in many material systems such as metals, ceramics and even proteins[1-5]. Because of crystal symmetry breaking during the transformation, multiple degenerate, crystallographically equaverlent martensiticvariants form self-accommodating, long-range ordered domain patterns[2] that underpin the "smart" properties of a ferroelasic system such as shape memory effect (SME) and superelasticity (SE). Crystalline defects (such as point defects, dislocations, precipitates and grain boundaries) have been utilized to alter the transformation pathways and domain structures[6, 7]in ferroelastic systems to tailor their properties[8-10], such as the temperature range of SE and size of hysteresis.Recently, a non-conventional transformation pathway (i.e., strain glass transition) that leads to nanoscale strain domain patterns was reported in TiNi [7, 11-13], Ti-Pd-Cr[14], Au-Cu-Al[15] and $\mathrm{TiNb}[16]$ alloys through defect engineering (e.g., by introducing anti-site and interstitial point defects and nano-precipitates). Different from the well studied "precursor" phenomenon (i.e., tweed) [17-20], which is described as a metstable state above the MT start temperature, $\mathrm{M}_{\mathrm{s}}$, this strain glass state can be regarded as a "frozen" precursory state [21] and the system does not transform into normal polytwinned martensite with long-range strain ordering (i.e., a strain crystal) under further cooling. The strain glass (STG) systems are found to have unique properties that are absent in normal martensitic systems, including continuoustransformation characteristics, frequency dispersion of storage modulus, 
slim hysteresis and low modulus $[7,10,13,15,16,21-28]$. The discovery of this unique strain glass transition (STGT) pathway not only opens a new avenuefor theoretical study of differentstrain states and their distinct properties in ferroelastic systems, but may also lead to the development of new alloys for noval applications in differentfields[14, 26, 29].

Since the discovery of the STGT, different models have beenproposed[25, 30-34] to explain the physical origin of the STG state. It was shown thatlocal lattice distortion created by point defects plays an important role in changing the transformation pathway from a sharp first-order MT to a continuous STGT and producing nanosized martensitic domains without long-range strain ordering in doped ferroelastic systems.However, experimental observations have shown that not all such defectscan change a normal MT to a STGT[5]. Up to now, the STGT has been observed in ferroelastic systems with only limited martensitic structures through doping specific defects. In TiNi-based ferroelastic systems, for example, only specific elements doping (such as excess $\mathrm{Ni}, \mathrm{Fe}, \mathrm{Cr}, \mathrm{Mn}$, but not $\mathrm{Cu}$ and $\mathrm{Pt}$ ) can produce theSTG states[12, 13, 28] and the reported STG states are all R(trigonal)-phase STGs (i.e., R-phase nanodomains) [11-13, 28]. There is no report of B19or B19'STGyetin doped TiNi-based ferroelastic systems, even though B19or B19' is the most stable martensitic phase found in these alloy systems. Comparing with the small stress-free transformation strain (SFTS) values of the $\mathrm{R}$ phase $(<1 \%)$, a potential B19 or B19'STGmay show much wider applications because of their giantSFTS (>8\%). 
Although there is no doublt that random fields from stress-carrying defects are necessary to create the STG state, a critical "strength" of the stress-carrying defects should exist in a ferroelastic crystal that deterimines whether its strain state is a strain crystal or a strain glass upon cooling. Such a critical strenghmay be measured by the relative "strength" of the randam strain (lattice distorion) field created by the defects to the transformation strain that has to be accommodated during MTs.Previous studies $[25,31,33,34]$ have focusedmainly on the effect of defect concentration on the formation of STG. In this study, we investigate the effect of defect strength at a fixed defect concentration on the transition behavior of a dopedferroelasticsystem. This may explain the experimentallyobserved phenomenon that when martensitic systemsare doped with the same amountbut different types of point defects, some of them become STG systems and some of them remain as martensitic systems.In particuilar we identify such critical defect strength using three-dimensional(3D) phase field simulations[23, 30, 35, 36] based on Landau theory of phase transformations[3, 37], gradient thermodynamics[38] and microelasticity theory of two-phase coherent solids[39] in an attempt to address the following key questions concerning STGs: why there is no B19 or B19'STG states in TiNi-based alloys by impurity doping andwhat kind of defects can change the normal B19 or B19' strain crysal (normal martensite) to the corresponding STG.

To measure the relative defect strength, we introduce a defect strength coefficient, $\phi$, which is the ratio between the equivalent Von Mises strainof the eigenstrain of a given type of defects and the equivalent Von Mises strain of the stress-free 
transformation strain (SFTS) tensor of a given type of MT. A new phase diagram connecting temperature with defect strength coefficient $\phi$ is established, which describeshow the $\phi$ value alters strain state in NiTi systems. Such a phase diagram may guide defect engineering to develop new STGs of larger transformation strains for broader applications. The simulation results also show that for a symmetrical anti-site defect, the critical defect strength coefficient, $\phi_{c}=8.9$ is necessary to produce a B19 strain glass.

\section{Model}

The model systems considered are generic single crystals undergoing improper cubic $\rightarrow$ trigonal $(\mathrm{R})$ andcubic $\rightarrow$ orthorhombic (B19) martensitic transformations, which have relatively small and large transformation strains respectivelyin NiTi-based shape memory alloys (SMAs).

The Local chemical free energy of the systems is approximated by a sixth-order Landau polynomial typical for improper MTs[35, 40]. The simplest form of such a polynomial reads,

$$
f_{c h}\left(\eta_{1}, \cdots \eta_{n}\right)=\frac{1}{2} A_{1} \sum_{i=1, \cdots n} \eta(\mathbf{r})_{i}^{2}-\frac{1}{4} A_{2} \sum_{i=1, \cdots n} \eta(\mathbf{r})_{i}^{4}+\frac{1}{6} A_{3}\left(\sum_{i=1, \cdots n} \eta(\mathbf{r})_{i}^{2}\right)^{3}
$$

where $\eta_{i}(i=1-n)$ are structural order (SO) parameters that characterize the symmetry-related $n$-fold degnerate transformatoin pathways (or corespondance variants of martensite). $n$ is determined by the symmetry of both the austenitic and martensitic phases. For a parent phase with a cubic symmetry, the $n$ valuses for 
different martensitic phases including R, O/B19 and B19' are 4, 6 and 12 respectively[2, 41].The SFTS tensor, $\varepsilon_{i j}^{M}(p)(p=1-n)$, for each of the $n$-fold degenerate correspondance variants of martensite can be formulated according to the lattice parameters of austenite and martensitic phases and the lattice correspondence between them[2, 41].The total strain at point ris given by $\varepsilon_{i j}(\mathbf{r})=\sum_{p=1}^{n} \varepsilon_{i j}^{M}(p) \eta(\mathbf{r})_{p}^{2}$, where, $\varepsilon_{i j}^{M}(p)$ is the tensor of the transformation strain and $n$ is the number of martensitic variants. Thus the crystal structure at the pointr is represented by the value of the order parameters, e.g., $\left(\eta_{1}, \eta_{2}, \cdots, \eta_{n}\right)=(0,0, \cdots, 0)$ represents the austenite (cubic)and $\left(\eta_{1}, \eta_{2}, \cdots, \eta_{n}\right)=\left(\eta_{0}, 0, \cdots, 0\right),\left(0, \eta_{0}, \cdots, 0\right), \cdots$, or $\left(0,0, \cdots, \eta_{0}\right)$ represents one of the martensitic variants, where $\eta_{0}$ is the equilibrium value ofthe structural order parameter.

In Eq. (1) $A_{1}=A_{1}^{0} \cdot\left(T-T^{0}(\mathrm{c})\right), \mathrm{A}_{1}^{0}, \mathrm{~A}_{2}, \mathrm{~A}_{3}$ and $\mathrm{A}_{4}$ are positive constants, $\mathrm{c}$ is average defect concentration in the simulations, $T^{0}(\mathrm{c})=T^{00}+b \cdot \mathrm{c} \quad\left(T^{00}=300, \mathrm{~b}=-300\right.$, and $\mathrm{c}=0.03$ ), and $b$ characterizes the relative strength of the global transition temperature effect (GTTE) associated with point defects[25].The GTTE describes how the doped anti-site defects alter the average thermodynamic stability of martensite and change the transition temperature of the whole system. We assume that itdoes not create spatial variation of the Landau potentional.

In contrast, the local stress field effect (LFE) associated with local lattice distortions $[25,42], \varepsilon_{i j}^{\text {local }}(\mathbf{r})$, caused by the point defects creates spatial variatoin and symmetry-breaking of the Landau potential[25]. The excess energy associated with 
the LFE can be described by the following equation[23, 25]

$$
f_{L}(r)=C_{i j k l} \sum_{i, j, k, l=1,2,3} \varepsilon_{i j}^{l o c a l}(\mathbf{r}) \cdot \varepsilon_{k l}^{M}(\mathbf{r})
$$

where $\varepsilon_{k l}^{M}(\mathbf{r})$ describes the SFTS of the martensite phase.

Note that both strains and shuffles are two important modes of the MTs considered in this study. Since the focus of the current work is on the effect of interplay between the transformation strain and the eigenstrain of defects, and the transformation strain is independent of shuffle, the shuffle modes are ignored in this paper.

The non-local gradient energy, $f_{g r}\left(\eta_{1}, \cdots \eta_{n}\right)$, associated with spatial non-uniformity of the SO parameters[38], can be aproximated by

$$
f_{g r}\left(\eta_{1}, \cdots \eta_{n}\right)=\frac{1}{2} \beta\left(\sum_{i=1, \cdots n}\left(\nabla \eta_{i}\right)^{2}\right)
$$

where $\beta$ is the gradient energy coefficient;

The coherency elastic strain energy, $E_{e l}$, associated with the lattice mismatch between the austenite and martensite and among different variants of the martensitic phase, formulated based on Khachaturyan's microelasticity theory[30, 39], is given by

$$
\begin{aligned}
E_{e l} & =\frac{1}{2} C_{i j k l} \sum_{p=1}^{n} \sum_{q=1}^{n} \varepsilon_{i j}^{M}(p) \varepsilon_{k l}^{M}(q) \int \eta_{p}^{2}(\mathbf{r}) \eta_{q}^{2}(\mathbf{r}) d^{3} r \\
& -\frac{1}{2} \sum_{p=1}^{n} \sum_{q=1}^{n} \int \frac{d^{3} k}{(2 \pi)^{3}} B_{p q}\left(\frac{\mathbf{k}}{k}\right)\left\{\eta_{p}^{2}(\mathbf{r})\right\}_{k}\left\{\eta_{q}^{2}(\mathbf{r})\right\}_{k}^{*},
\end{aligned}
$$

where $C_{i j k l}$ is the elastic modulus tensor, $\mathbf{k}$ is the wave vector defined in the reciprocal space, $\left\{\eta_{p}^{2}(\mathbf{r})\right\}_{k}$ is the Fourier transform of $\eta_{p}^{2}(\mathbf{r}),\left\{\eta_{p}^{2}(\mathbf{r})\right\}_{k}^{*}$ is the conjugate of 
$\left\{\eta_{p}^{2}(\mathbf{r})\right\}_{k}$, and the kernel $B_{p q}(\mathbf{k} / k)$ is $B_{p q}(\mathbf{e})=e_{i} \sigma_{i j}^{0}(p) \Omega(\mathbf{e}){ }_{j k} \sigma_{k l}^{0}(q) e_{l}$, where $\mathbf{e}=\mathbf{k} / k, \sigma_{i j}^{0}(p)=C_{i j k l} \varepsilon_{i j}^{M}(p), \Omega(\mathbf{e})_{i j}^{-1}=C_{i j k l} e_{k} e_{l}$. The SFTS of the martensitic phase can be described in terms of the order parameter fields, i.e., $\varepsilon_{i j}=\sum_{p=1}^{n} \varepsilon_{i j}^{M}(p) \eta_{p}^{2}$.

The total free energy of the system, $F$, consists of all the above contributions, i.e.,

$$
F=\int d^{3} \mathbf{r}\left[f_{c h}\left(\eta_{1}, \cdots \eta_{n}\right)+f_{L}+f_{g r}\left(\eta_{1}, \cdots \eta_{n}\right)\right]+E_{e l},
$$

and its variational derivatives with respect to the order parameter fields form the thermodynamic driving force for microstructure evolution during transitions among different strain states (i.e., austenite, martnesite and strain glass) characterized by the order parameters, following the stochastic time-dependent Ginsburg-Landau equation:

$$
\frac{d \eta_{p}(\mathbf{r}, t)}{d t}=-M \frac{\delta F}{\delta \eta_{p}(\mathbf{r}, t)}+\xi_{p}(\mathbf{r}, t), \quad p=1, \cdots, n
$$

where $\xi$ is the Langevin noise term describing thermal fluctuations and $M$ is the dynamic coefficient.

To compare two different strain tensors (defect distortion and SFTS of martensite), we define a defect strength coefficient $\phi$, which can be calculated as the ratio of the equivalent Von Mises strain caused by defect and the equivalent Von Mises strain caused by the martensitic phase transformation, i.e., $\phi=\frac{\left|\varepsilon_{e q}^{\text {defect }}\right|}{\left|\varepsilon_{e q}^{S F T S}\right|}$, where the equivalent Von Mises strain can be calculated as $\varepsilon_{e q}=\frac{2}{3} \sqrt{\frac{3\left(\varepsilon_{11}^{2}+\varepsilon_{22}^{2}+\varepsilon_{33}^{2}\right)}{2}+\frac{3\left(\varepsilon_{12}^{2}+\varepsilon_{23}^{2}+\varepsilon_{13}^{2}\right)}{4}}$. For example, consider an anti-side defect in NiTi, where an additional $\mathrm{Ni}$ atom occupies theTi-sublaticein a B2 unit cell and 
changesit to a BCC unit cell of Ni (Fig. 1). This will create a dilational strain with a maximum value: $\varepsilon^{\text {defect }}=\left(\begin{array}{ccc}-0.075 & 0 & 0 \\ 0 & -0.075 & 0 \\ 0 & 0 & -0.075\end{array}\right)$ (i.e., $\left.\frac{a_{b c c}^{N i}-a_{B 2}^{N i T i}}{a_{B 2}^{N i i}}\right)$ withVon Mises strain $\varepsilon_{\text {eq }}^{\text {defect }}=0.1061$.According to the SFTS of the R phase[23, 43], i.e., $\varepsilon^{R}=\left(\begin{array}{ccc}-0.000246 & 0.006038 & 0.006038 \\ 0.006038 & -0.000246 & 0.006038 \\ 0.006038 & 0.006038 & -0.000246\end{array}\right)$, the equivalent Von Mises strain is $\varepsilon_{e q}^{R}=0.01207$, and we have the coefficient $\phi_{R}^{N i}=\frac{\varepsilon_{e q}^{\text {defect }}}{\varepsilon_{e q}^{R}}=8.76$ for Ni doping.In the present work, we change the value of lattice distortion of $\varepsilon^{\text {defect }}$ and alter the coeffieicnet $\phi$ to determine the critical value of defect strength in the formation of strain glass. The dimensionless materials parameters [23] used in our simulations are listed in Table. 1 .The system size considered is $64 \mathrm{~nm} \times 64 \mathrm{~nm} \times 64 \mathrm{~nm}$ with a $64 \times 64 \times 64$ numerical mesh and periodical boundary conditions are applied along all three dimensions.Need to note, we assume that the phase field voxels which contain defects produce a distortion strain $\varepsilon^{\text {defect }}$ in our simulations, and the dimensionless concentration $c$ describe the volume fraction of phase field voxels including defects.Thedimensionless defect concentrationis fixed to $c=0.03$ in our simulations.

\section{Results}

\subsection{Critical defect strength for the formation of $\mathbf{R}$ strain glasses}

Fig. 2 shows the calculated volume fraction of $\mathrm{R}$ martensitetransition upon 
cooling and heating with different defect strength coeffieicnet $\phi$. When $\phi=0.0$ (defect free), Fig.2 (a) shows typical first order transition characteristics (i.e., sharp change of the volume fraction at the transition temperature) upon cooling and large temperature hysteresis under thermal cycling. When the defect strength $\phi<7.0$ (Figs. 2 (b) -(d)), the volume fraction changeis still sharp and the temperature hysteresis under thermal cycling is still obvious but become smaller and smaller. When $\phi \geq 7.0$, the systems show gradual change of the volume fraction with almost zero temperature hysteresis as shown in Figs. 2 (e-f). These results show that increasing defect strength at fixed defect concentration cansuppress normal MT and change it to a continuous transition.

Fig. 2(g) sumarizes the variation of temperature hysteresis as functin of defect strength coefficient $\phi$, showing a continued descrease of the hysteresis as $\phi$ increases and, when $\phi \geq 7.0$, the hysteresis vanishes. The vanishing of hystersis in temperature cycle implies a continuous transition, and this special defect strength coefficient can be defined as the critical value of $\mathrm{R}$ strain glass transition (i.e., $\phi_{c}=7.0$ ) in the model system. Note that the critical defect strength coefficient $\phi_{c}$ varies with defect concentration, as shown in Fig. 3. The lower is the defect concentration, the higher is the defect strength needed to produce a strain glass state. This result are consistent with the experimental observations that different dopants (such as $\mathrm{Ni}$ and $\mathrm{Fe}$ anti-site defects) require different critical defect concentrations for the R strain glass transition, e.g., $\mathrm{Ti}_{48.5} \mathrm{Ni}_{1.5}[28]$ and $\mathrm{Ti}_{50} \mathrm{Ni}_{44} \mathrm{Fe}_{6}[13]$. In excess $\mathrm{Ni}$ doped NiTi system, the defect strenth coefficient $\phi_{R}^{N i}=8.76$, which is larger than the critical value $\phi_{\mathrm{c}}=7.0$, so the system can become an R strain glass with excess Ni doping. 
Based on the temperature dependence of volume fraction of martensite shown in Figs. 2(a)-(f), we establish a temperature vs. defect strengthphase diagram(Fig. 4) with fixed defect concentration $\mathrm{c}=0.03$. In this phase diagram, $\mathrm{T}_{\mathrm{S}}$ describes the start temperature of martensitic phase transformation or strain glass transition, and $\mathrm{T}_{\mathrm{F}}$ describes the finish temperature of thesetransitions. $T_{S}$ isdefined as the temperature at which the volume fraction of martensite reaches $5 \%$ and $\mathrm{T}_{\mathrm{F}}$ is defined as the temperature at which the volume fraction of martensite reaches $90 \%$. The phase diagram indicates that the gap between $T_{S}$ and $T_{F}$ increases when the defect strength increases. In addition, $\mathrm{T}_{\mathrm{S}}$ show almost a linear increasewith defect strength, which indicates that the defect strength defined in the current study represent reasonabley well the potency of the anti-site defects for inducing martensitic domains.In contrast, $\mathrm{T}_{\mathrm{F}}$ shows a non-monotonic dependence on the defect strength. Although the anti-site defects promote nucleation of martensite, they suppress autocatalysis and confine the growth when their strength becomes high enough because of their randomness in space.

The insets in Fig. 4 describe the corresponding microstructural states at different defect strength and temperature, where red, blue, yellow and green represent the four variants of the $\mathrm{R}$ martensitic phase, and the austenite is transparent. Clearly as the defect strength increases the self-accomodating, long-range ordered twinning martenste changes gradually into randomly distributed nano-domains of individual martensitic variants. In between $\mathrm{T}_{\mathrm{S}}$ and $\mathrm{T}_{\mathrm{F}}$, there exist precusory nanodomains (tweed)and, below $\mathrm{T}_{\mathrm{F}}$, the system transforms either to well-defined, internally 
twinnedmartensite when the defect strength $\phi<3.5$ or to a strain glass (identified by the vanished temperature hysteresis shown in Fig. 2) when defect strength $\phi \geq 7.0$ (i.e., $\left.\phi_{c}\right)$.Note that the transition from a strain crystal to a strain glass when defect strength increase israther diffuse.

\subsection{Critical defect strength for B19 strain glass}

The above simulation results show that the critical defect strengthfor the formation of $\mathrm{R}$ strain glass is $\phi_{c}=7.0$, which is less than the maximum value of $\phi_{R}^{N i}=8.76$ when excess $\mathrm{Ni}$ replaces $\mathrm{Ti}$ and forms anti-site defects in NiTi. This explains why experimental observationshave shown R strain glassesin NiTi-based ferroelastic systems. In order to understand why there is no B19/B19' strain glass discovered in TiNi-based alloys, we calculate the critical defect strength for B19 strain glass transition in the same system (defect concentration is also fixed to 0.03). Note that we choose B19 not B19' because B19 have less number of martensitic variants which may save the simulaiton time. The typical SFTS of B19 is used $\varepsilon^{B 19}=\left(\begin{array}{ccc}0.02915 & 0 & -0.02915 \\ 0 & -0.0421 & 0 \\ -0.02915 & 0 & 0.02915\end{array}\right)$ [2] and its eigenvalues are $-0.0421,0$, and

0.0583. Thus we have $\varepsilon_{e q}^{B 19}=0.0587$ and we have defect strength coefficient $\phi_{B 19}^{N i}=\frac{\varepsilon_{e q}^{\text {defect }}}{\varepsilon_{e q}^{B 19}}=1.27(\mathrm{Ni}$ replace $\mathrm{Ti})$ and $\phi_{B 19}^{C u}=\frac{\varepsilon_{e q}^{\text {defect }}}{\varepsilon_{e q}^{B 19}}=0.71(\mathrm{Cu}$ replace $\mathrm{Ni})$. The calculated volume fraction change upon cooling and heatig for different defect strength coefficients $\phi$ are shown in Fig. 5. The results show that the temperature hysteresis changes to a constant (close to zero) when the defect strength $\phi \geq 8.9$ (Fig. 5 
(g)). Thus the critical defect strength coefficient for the formation of a B19 strain glassis $\phi_{c} \sim 8.9$.Fig. 5(h) shows the corresponding microstructures of B19 martensite obtained at different defect strength.

The above simulation results show that bothB $2 \rightarrow \mathrm{R}$ and $\mathrm{B} 2 \rightarrow \mathrm{B} 19$ martensitic transformations can be suppressed into strain glass transitions whenthe defect strenth $\phi$ is larger than a critical value, e.g., $\phi_{c}=7.0$ for R strain glass and $\phi_{c}=8.9$ for B19 strain glass. However, the B19 martensite has much larger enginvalues of the SFTS and thus the Von Mises strain. The formation of B19 strain glass would require a much larger value of the dilational strain from an anti-site defect, e.g., $\varepsilon_{e q}^{\text {defect }} \cong 0.52$ according to the definition of the defect strength coefficient $\phi$ introduced earlier), which is impossible for point defects doping. This may explain whythere is no B19 strain glass found in TiNi-based systems.

As mentioned earlier, it is the interplay between self-accommodation among different martensitic variants and random fields associated with stress-carrying defects that determines the transformation paths leading either to a strain crystal or to a strain glass in a ferroelastic system upon cooling. To characterize such an interplay we plot the relationship between the ratio of defect-martensite interactoin energy and martnesite-martensite interaction energy, $\frac{E_{M-D}^{\max }}{E_{M-M}^{\max }}$,obtained from the phase field simulations and defect strength coefficient $\phi$ in Fig. 6. $E_{M-D}^{\max }$ describes the maximum interaction energy between a single anti-site defect and a martensitic variant, and $E_{M-M}^{\max }$ describes the maximum interaction energy between different martensitic 
variants. $\phi_{C}^{R}$ and $\phi_{C}^{B 19}$ are the critical defect strength coefficients for $\mathrm{R}$ strain glass and B19 strain glass, respectively, according to Fig. 1 and Fig. 5. At the critical defect strength for $\mathrm{B} 19$ strain glass, $\phi_{C}^{B 19}, \frac{E_{M-D}^{\max }}{E_{M-M}^{\max }}=16$. This indicates that the defect-martensite interaction energy must be sixteen times of the martensite--martensite interaction energy in order to produce a B19 strain glass state (at this concentration 0.03). Note that this critical value of the defect-martensite interaction energy will be different when the defect concentration changes. A higher defect concentration in general will require lower defect-martensite interaction energy.

\section{Discussions}

The above simulation results suggest the existance of a critical defect strength $\phi$ to induce a strain glass transition in doped ferroelastic systems and explain why B19 strain glass is difficult to form in the NiTi system. In this part, we try to explore the physical origin through calculating the extra interaction caused by defect (by using R strain glass as example).Fig. 7 shows the local stress field caused by a single anti-site defect in the centre of a simulation cell of $64 \mathrm{~nm}$ cube under periodical boundary condition in all three dimensions. The local stress field was calculated using Khachaturyan's microelasticity theory[39] by using the eigenstrain of the anti-site defect (as illustrated in Fig. 1). Figs. 7(a)-(f) show the stress field distribution of $\sigma_{11}$, $\sigma_{22}, \sigma_{33}, \sigma_{12}, \sigma_{13}$ and $\sigma_{23}$, respectively, around the anti-site defect, where the purple and light blue colors describe the isosurfaces of the tensile and compress stress fields, 
respectively. The existence of stress field will produce different interactions between the defect and different martensitic variants and will influence the microstructural state. Fig. 7(g) shows the negative excess interaction energy calculated for different martensitic variants inthe local stress field of the anti-site defect. It shows that this anti-site defect preferscertain martensitic variants (nagtive values) and supress some others(positive values) at different locations. Because the anti-site defects are randomly distributed in space, the interaction energy fields are random as well, causing the thermodynamic frustration.

The change of defect strength affects directly the amplitudes of the local stress fields and, hence, the degreee of the frustration.Fig. 8(a) and (b) show the stress amplitude distributions (potency distribution) of local stress fields $\sigma_{11}$ and $\sigma_{12}$ created by the anti-site defects in $\mathrm{Ti}_{48.5} \mathrm{Ni}_{51.5}$ obtained from the phase field simulations with different defect strength coefficient $\phi$. Such a distribution is calculated through counting the numbers of volume elements that have the same local stress value within a given defect strength coefficient in the entire system. Because our simulations use symmetrical anti-site defects and their eigenstrains are purely dilatational, the other stress components $\left(\sigma_{22}, \sigma_{23}, \sigma_{33}, \sigma_{13}\right)$ should have similar amplitude distributions as either that of $\sigma_{11}$ or that of $\sigma_{12}$. The calculation results show that the stress amplitude distribution becomes broader when the defect strength coefficient $\phi$ increases. The broader stress amplitude distribution will produce more influence area with high interaction as shown in Fig. 8(c) and prevent the formation of ordered ground state (twinned M). Instead, the system forms a glassy state (i.e., randomly distributed 
nanodomains of individual variants of martensite). The broad distribution of activation energy due to contributions from interaction energy caused by defects (i.e., potency distribution of the defects) as shown in Fig. 8 (c) is the key effect of the continuous strain glass transition process in doped ferroelastic systems. This broad distribution of activation energy will lead to a broad martensite nucleation temperature and smooth transition. Fig. 9 (a) shows the spatial distribution of phase field voxels containing defects in the system, Figs. 9(b)-(f) showsthe spatial distribution of Local Von Mises stress $\left(\sigma_{\mathrm{VM}}\right)$ caused by defects with different defect strength coefficient $\phi$, where, Local Von Mises stress can be calculated: $\sigma_{V M}=$ $\sqrt{\frac{\left(\sigma_{11}-\sigma_{22}\right)^{2}+\left(\sigma_{11}-\sigma_{33}\right)^{2}+\left(\sigma_{22}-\sigma_{33}\right)^{2}+6\left(\sigma_{12}^{2}+\sigma_{23}^{2}+\sigma_{13}^{2}\right)}{2}}$. With the increase of defect strength coefficient $\phi$, the local stress distribution caused by defects becomes more inhomogeneous. Simultaneously, the volume of high stress regions (green and yellow color) increase and these regions are separated by low stress regions (blue color) when defect strength coefficient is high (see Fig. 9(e) and (f)). High stress regions prefer the nucleation of $\mathrm{M}$ domains but the local stress regions limits the growth. The randomly distributed local stress field influences the nucleation and growth of martensitic domains and should be the physical origin of strain glass transition.

The change of the total free energy of the systems ( $R$ martensite and $\mathrm{R}$ strain glass) upon cooling calculated for different defect strengths are shown in Fig. 10. $\phi=0.0$ and $\phi=3.5$ describing the free energy changesaccompanying normal martensitic transformations(with the formation of long range ordered, internally twinned martensitic plates) and $\phi=7.0$ and $\phi=8.76$ describing the free energy 
changesaccompanying thestrain glass transitions(with the formation of nanosized martensitic domains). Fig 10 (a) shows the changes of the local chemical free energy, $F_{\text {ch }}$, calculated according to Eq.(1). All the chemical free energy curves with different defect strengths show a decrease upon cooling. The chemical free energy curves of $\phi=0.0$ and $\phi=3.5$ show a sudden change at transition temperature, while the chemical free energy curves of $\phi=7.0$ and $\phi=8.76$ show a gradual change upon cooling. Furthermore, the chemical free energy curves show almost the same driving force (i.e., the free energy difference between the highest and lowest temperatures)upon cooling for both normalmartensite $(\phi=0.0$ and $\phi=3.5)$ and strain glass $(\phi=7.0$ and $\phi=8.76)$. The chemical free energy of strain glass is a little higher than that of the internally twinnedmartensite because there exist more domain boundaries in the strain glass system. Figs. 10 (b) and (d) show the non-local gradient energy, $F_{\text {gr }}$ (Eq. (3)), and the coherency elastic strain energy, $F_{\mathrm{el}}($ Eq. (4)), respectively.Both gradient energy and elastic strain energy show an increase upon cooling with different defect strength coefficients. The gradient energy and elastic strain energy curves obtained for $\phi=0.0$ and $\phi=3.5$ show small increases with the formation of long range ordered, internally twinnedmartensite upon cooling, while the gradient energy and elastic strain energy curves for $\phi=7.0$ and $\phi=8.76$ showhuge increaseswith the existence of randomly distributed nanosized martensitic domains. The long range ordered, internally twinned martensitic states (in $\phi=0.0$ and $\phi=3.5$ ) include a low density of domain boundaries with atypical twin structure, which results in the minimum elastic strain energy and gradient energy.On the contrary, the glassy martensitic states(in $\phi=7.0$ and $\phi=8.76$ ) 
include irregular shaped martensitic nanodomainswith alarge density of domain boundaries, which increase both the elastic strain energy and gradient energy. In the view of elastic energy and gradient energy, strain glass state (with high $\phi$ ) should be unstable because it includes much higher energy than that of twin martensite (with low $\phi$ ). However, the fact is different (i.e., strain glass state with nanosized martensitic domains is stable when $\phi=7.0$ and $\phi=8.76$ ), defects must produce extra interaction in stabilizing this disorder state. Fig. 10 (c) shows the excess free energy $F_{\mathrm{L}}$ (i.e., the extra interaction energy) caused by point defects according to Eq. (2). The excess free energy shows a decrease upon cooling when $\phi$ is not equal to zero. The excess free energy of $\phi=3.5$ show a small decrease with sudden change, while the excess free energy of $\phi=7.0$ and $\phi=8.76$ show a large decrease with gradual change. The excess energy caused by point defects provides an extra driving forceto compensate the high elastic energy and gradient energy and stabilize the system to a glassy martensitic state. Fig. 10 (e) shows the total free energyF $F_{\text {total }}$ (Eq. 5) of the whole system with different defect strength. The total free energy shows a decrease for both martensitic transition (in $\phi=0.0$ and $\phi=3.5$ ) and strain glass transition (in $\phi=7.0$ and $\phi=8.76)$ upon cooling. It describes that both the formation of nanodomains at $\phi=7.0$ and $\phi=8.76$ and the formation of twin martensite at $\phi=0.0$ and $\phi=3.5$ upon cooling are reasonable.It implies that strength of doped defects is the most important effect to change the phase transition pathway from first order martensitic transformation to continuous strain glass transition. To separate the effect of defect strength, we calculate the total free energy without the excess free energy $F_{L}$ as shown 
in Fig. 10(f). The free energy curves of $\phi=0.0$ and $\phi=3.5$ in Fig. 10 (f) don't show big difference from that of Fig. 10 (e),which describes that the extra interaction caused defects is too small to suppress normal martensitic transformation when the strength coefficient is small.Differently, the free energy curves of $\phi=7.0$ and $\phi=8.76$ in Fig. 10(f) have shown huge difference from that ofFig. 10 (e), which describes that the extra interaction causedby defects must be large enough to suppress the normal MT when the defect strength coefficient is larger than critical value.

To clarify the frustration effect of $F_{\mathrm{L}}$ caused by point defects, Fig. 11shows the free energy evolution with initial nanosized martensitic domain state (from $\phi=7.0$ ) and then evolve with removing the excess energy $F_{\mathrm{L}}$. The black dot shows the initial total free energywith the nanosized martensitic domain state at $\mathrm{T}=0.8 \mathrm{~T}_{0}$ and $\phi=7.0$. The blue circles describe the free energy change with time after removing the $F_{\mathrm{L}}$ (but keep the initial nanodomain microstructure) in our simulations(i.e., $\mathrm{T}=0.8 \mathrm{~T}_{0}$ and $\phi=0.0$ ). The free energy curve shows a decrease, which implies that nanosized domain state (strain glass state)willnot be stable any moreif there is no interaction caused by defects. Insets show related microstructural evolution with time. The microstructures evolutions show that the nanosized martensitic state will grow and change into long range ordered twin martensitic state (i.e., the ground state). Our calculationsconfirm that the value of interaction effects caused by $F_{L}$ play an key role in suppressing the long range ordered martensite and stabilizing the nanosized martensitic domains, which depends on the defect strength coefficient. 
The competition between the interaction caused by defect and martensitic variants and theinteraction caused by different martensitic variants is the physical origin of frustration and the formation of glassy martensitic states in doped ferroelasticsystems. Small lattice distortion caused by certain defects may produce too weak interaction tofrustratenormal twinned martensitic state with large SFTS. The equivalent Von Mises strain of STFS of B19 phase is about six times larger than that of R phase, which results in a larger driving force for the formation of B19 twin martenstic state[5]. So a largerdefect strain caused by point defects is needed to produce large enough extra interacrtion than that of R phase.Similarly, B19' phase transition has a larger value ofeiginvalue of STFSthan that of B19, which means that the formation of B19'nanodomains also need much larger value of the lattice distortion strain. Our simulationsalso imply that the doped anti-site defects $(\mathrm{Ni}, \mathrm{Fe}, \mathrm{Cu} . .$.$) in TiNi-based$ alloys will be difficult to produce such a large lattice distortion ( $\varepsilon_{e q}^{\text {defect }} \square 0.5$ )to produce B19/B19'strain glass, other stress-carrying defects (e.g., precipitates, dislocations, grain boundaries) which can produce larger extra interaction must be created to produce this kind of strain glass. Dislocation loops which can produce a Von Mise strain $\sim 0.5$ will be a posible way to influence normal MT[44] and produce B19/B19' strain glass. In addition, recently experiments show that nano-grains with huge grain boundaries can confine the nucleation and growth of B19' Martensite and produce B19'strain glass[10]. Precursory phase decomposition with compositional and structural non-uniformity can also produce strain glass[22]. More work are needed in future to study relationship between different defect types and different 
martensitic phases.

\section{Summary}

In summary, we study the strain glass transition in doped ferroelastic systems through phase field simulations. The role of lattice distortion caused by stress-carrying defects are studied and a phase diagram including temperature and defect strength is established. A defect srength coefficient which describes the ratio between the equivalent Von Mises strain caused by defect and the equivalent Von Mises strain caused by the martensitic phase transformation is defined in our simulations. We show that the critical defect strength coefficient for the formaiotn of $\mathrm{R}$ glassy martensitic state at $\mathrm{c}=0.03$ is about $\phi=7.0$. When the defect strength coefficient $\phi$ is larger than this critical value, the system will be changed from normal first order martensitic transition with large hysteresis into continuous strain glass transition with small hysteresis. Thefree energy calculations show that the value of excess energy caused by doped point defects play key role in suppressing the long range ordered martensitic twin and stabilizing the glassy martensitic state. Through calculating the critical defect strength's effect for both R phase and B19 phase, our simulations implys that the formation of B19 strain glass also need a larger critical defect strength coefficient i.e., $\phi=8.9$. Considering the larger eiginvalue of SFTS of B19, equivalent Von Mises strain caused by defect must be much larger than that of $\mathrm{R}$ strain glass to form B19 strain glass. This explains the puzzle that why there is only strain glass with local R local structure (not B19 or B19') in TiNi-based ferroelastic 
systems. Our simulations may shed light on the development of new shape memory alloys with nanosized martensitic domains structure and small hysteresis through defect engineering using extended defects such as dislocations and grain boundaries rather than point defects.

\section{Acknowledgments:}

The work was supported by National Basic Research Program of China (2012CB619402, 2014CB644003and 2010CB631003), National Natural Science Foundation of China $(51201125,51471127,51071117,51171140)$, China Postdoctoral Science Foundation (2012M511996) and 111 Project of China (B06025); the U.S. Natural Science Foundation Grant No. DMR-1410322(Yunzhi Wang) and US Department of Energy Grant No.DE-SC0001258 (YipengGao); and the Japan KAKENHI (XiaobingRen).

\section{References:}

[1] K. Otsuka, C.M. Wayman, Shape memory materials, Cambridge University Press, Cambridge ; New York, 1998.

[2] K. Bhattacharya, Microstructure of martensite : why it forms and how it gives rise to the shape-memory effect, Oxford University Press, Oxford ; New York, 2003.

[3] E.K.H. Salje, Phase Transitions in Ferroelastic and Coelastic Materials, Cambridge University Press, Cambridge, 1990.

[4] K. Bhattacharya, S. Conti, G. Zanzotto, J. Zimmer, Crystal symmetry and the 
reversibility of martensitic transformations, Nature, 428 (2004) 55-59.

[5] K. Otsuka, X. Ren, Physical metallurgy of Ti-Ni-based shape memory alloys, Progress in Materials Science, 50 (2005) 511-678.

[6] Y. Gao, N. Zhou, F. Yang, Y. Cui, L. Kovarik, N. Hatcher, R. Noebe, M.J. Mills, Y. Wang, P-phase precipitation and its effect on martensitic transformation in $(\mathrm{Ni}, \mathrm{Pt}) \mathrm{Ti}$ shape memory alloys, Acta Materialia, 60 (2012) 1514-1527.

[7] Y.C. Ji, X.D. Ding, T. Lookman, K. Otsuka, X.B. Ren, Heterogeneities and strain glass behavior: Role of nanoscale precipitates in low-temperature-aged $\mathrm{Ti}_{48.7} \mathrm{Ni}_{51.3}$ alloys, Physical Review B, 87 (2013).

[8] R. Zarnetta, R. Takahashi, M.L. Young, A. Savan, Y. Furuya, S. Thienhaus, B. Maass, M. Rahim, J. Frenzel, H. Brunken, Y.S. Chu, V. Srivastava, R.D. James, I. Takeuchi, G. Eggeler, A. Ludwig, Identification of Quaternary Shape Memory Alloys with Near-Zero Thermal Hysteresis and Unprecedented Functional Stability, Advanced Functional Materials, 20 (2010) 1917-1923.

[9] J. Cui, Y.S. Chu, O.O. Famodu, Y. Furuya, J. Hattrick-Simpers, R.D. James, A. Ludwig, S. Thienhaus, M. Wuttig, Z.Y. Zhang, I. Takeuchi, Combinatorial search of thermoelastic shape-memory alloys with extremely small hysteresis width, Nature Materials, 5 (2006) 286-290.

[10] A. Ahadi, Q.P. Sun, Stress hysteresis and temperature dependence of phase transition stress in nanostructured NiTi-Effects of grain size, Applied Physics Letters, 103 (2013).

[11] S. Sarkar, X.B. Ren, K. Otsuka, Evidence for strain glass in the 
ferroelastic-martensitic system $\mathrm{Ti}_{50-\mathrm{x}} \mathrm{Ni}_{50+\mathrm{x}}$, Physical Review Letters, 95 (2005) 4.

[12] Y.M. Zhou, D.Z. Xue, X.D. Ding, Y. Wang, J.A. Zhang, Z. Zhang, D. Wang, K.

Otsuka, J. Sun, X.B. Ren, Strain glass in doped $\mathrm{Ti}_{50}\left(\mathrm{Ni}_{50-\mathrm{x}} \mathrm{D}_{\mathrm{x}}\right)(\mathrm{D}=\mathrm{Co}, \mathrm{Cr}, \mathrm{Mn})$ alloys: Implication for the generality of strain glass in defect-containing ferroelastic systems, Acta Materialia, 58 (2010) 5433-5442.

[13] D. Wang, Z. Zhang, J.A. Zhang, Y.M. Zhou, Y. Wang, X.D. Ding, Y.Z. Wang, X.B. Ren, Strain glass in Fe-doped Ti-Ni, Acta Materialia, 58 (2010) 6206-6215.

[14] Y.M. Zhou, D.Z. Xue, X.D. Ding, K. Otsuka, J. Sun, X.B. Ren, High temperature strain glass in $\mathrm{Ti}_{50}\left(\mathrm{Pd}_{50-\mathrm{x}} \mathrm{Cr}_{\mathrm{x}}\right)$ alloy and the associated shape memory effect and superelasticity, Applied Physics Letters, 95 (2009) 3.

[15] J. Liu, M. Jin, C. Ni, Y. Shen, G. Fan, Z. Wang, Y. Zhang, C. Li, Z. Liu, X. Jin, Strain glassy behavior and premartensitic transition in $\mathrm{Au}_{7} \mathrm{Cu}_{5} \mathrm{Al}_{4}$ alloy, Physical Review B, 84 (2011) 140102.

[16] Y. Nii, T. Arima, H.Y. Kim, S. Miyazaki, Effect of randomness on ferroelastic transitions: Disorder-induced hysteresis loop rounding in Ti-Nb-O martensitic alloy, Physical Review B, 82 (2010) 214104.

[17] S.M. Shapiro, J.Z. Larese, Y. Noda, S.C. Moss, L.E. Tanner, Neutron-Scattering Study of Premartensitic Behavior in Ni-Al Alloys, Physical Review Letters, 57 (1986) 3199-3202.

[18] D. Shindo, Y. Murakami, T. Ohba, Understanding precursor phenomena for the R-phase transformation in Ti-Ni-based alloys, MRS Bulletin, 27 (2002) 121-127.

[19] M.S. Choi, T. Fukuda, T. Kakeshita, Anomalies in resistivity, magnetic 
susceptibility and specific heat in iron-doped Ti-Ni shape memory alloys, Scripta Materialia, 53 (2005) 869-873.

[20] M.S. Choi, T. Fukuda, T. Kakeshita, H. Mori, Incommensurate-commensurate transition and nanoscale domain-like structure in iron doped Ti-Ni shape memory alloys, Philosophical Magazine, 86 (2006) 67-78.

[21] X.B. Ren, Y. Wang, Y.M. Zhou, Z. Zhang, D. Wang, G.L. Fan, K. Otsuka, T. Suzuki, Y.C. Ji, J. Zhang, Y. Tian, S. Hou, X.D. Ding, Strain glass in ferroelastic systems: Premartensitic tweed versus strain glass, Philosophical Magazine, 90 (2010) $141-157$.

[22] J.P. Liu, Y.D. Wang, Y.L. Hao, Y.Z. Wang, Z.H. Nie, D. Wang, Y. Ren, Z.P. Lu, J.G. Wang, H.L. Wang, X.D. Hui, N. Lu, M.J. Kim, R. Yang, New intrinsic mechanism on gum-like superelasticity of multifunctional alloys, Scientific Reports, 3 (2013).

[23] D. Wang, S. Hou, Y. Wang, X. Ding, S. Ren, X. Ren, Y. Wang, Superelasticity of slim hysteresis over a wide temperature range by nanodomains of martensite, Acta Materialia, 66 (2014) 349 - 359.

[24] D. Wang, Y. Ni, J. Gao, Z. Zhang, X. Ren, Y. Wang, Unique properties associated with normal martensitic transition and strain glass transition: A simulation study, Journal of Alloys and Compounds, 577 (2012) S102 -S106.

[25] D. Wang, Y. Wang, Z. Zhang, X. Ren, Modeling Abnormal Strain States in Ferroelastic Systems: The Role of Point Defects, Physical Review Letters, 105 (2010) 205702. 
[26] Y. Wang, X. Ren, K. Otsuka, Shape Memory Effect and Superelasticity in a Strain Glass Alloy, Physical Review Letters, 97 (2006) 225703.

[27] J. Zhang, Y. Wang, X.D. Ding, Z. Zhang, Y.M. Zhou, X. Ren, K. Otsuka, J. Sun, M. Song, Stress-induced strain glass to martensite (R) transition in a $\mathrm{Ti}_{50} \mathrm{Ni}_{44.5} \mathrm{Fe}_{5.5}$ alloy, Physical Review B, 83 (2011) 174204.

[28] Z. Zhang, Y. Wang, D. Wang, Y.M. Zhou, K. Otsuka, X.B. Ren, Phase diagram of $\mathrm{Ti}_{50-\mathrm{x}} \mathrm{Ni}_{50+\mathrm{x}}$ : Crossover from martensite to strain glass, Physical Review B, 81 (2010).

[29] T. Kakeshita, T. Fukuda, A. Saxena, A. Planes, Disorder and strain-induced complexity in functional materials, Springer, Heidelberg ; New York, 2012.

[30] S. Semenovskaya, A.G. Khachaturyan, Coherent structural transformations in random crystalline systems Acta Materiialia, 45 (1997) 4367 - 4384.

[31] P. Lloveras, T. Castan, M. Porta, A. Planes, A. Saxena, Influence of Elastic Anisotropy on Structural Nanoscale Textures, Physical Review Letters, 100 (2008) 165707.

[32] D. sherrington, A simple spin glass perspective on martensitic shape-memory alloys J.Phys.:Condens. Matter, 20 (2008) 304213.

[33] R. Vasseur, T. Lookman, Effects of disorder in ferroelastics: A spin model for strain glass, Physical Review B, 81 (2010) 094107.

[34] R. Vasseur, D. Xue, Y. Zhou, W. Ettoumi, X. Ding, X. Ren, T. Lookman, Phase diagram of ferroelastic systems in the presence of disorder: Analytical model and experimental verification, Physical Review B, 86 (2012).

[35] Y. Wang, A.G. Khachaturyan, Three-dimensional field model and computer 
modeling of martensitic transformations, Acta Materialia, 45 (1997) 759-773.

[36] Y.Z. Wang, J. Li, Phase field modeling of defects and deformation, Acta Materialia, 58 (2010) 1212-1235.

[37] L. D. Landau, and E. M. Lifshitz, Statistical Physics, Third Edition, 1980.

[38] J.W. Cahn, J.E. Hilliard, Free Energy of a Nonuniform System .1. Interfacial Free Energy, Journal of Chemical Physics, 28 (1958) 258-267.

[39] A. G. Khachaturyan, Theory of Structural Transformations in Solids, John Wiley \& Sons, New York, 1983.

[40] Y. Wang, A.G. Khachaturyan, Multi-scale phase field approach to martensitic transformations, Materials Science and Engineering: A, 438-440 (2006) 55-63.

[41] K. Bhattacharya, R.V. Kohn, Symmetry, texture and the recoverable strain of shape-memory polycrystals, Acta Materialia, 44 (1996) 529-542.

[42] D. Wang, X.Q. Ke, Y.Z. Wang, J.H. Gao, Y. Wang, L.X. Zhang, S. Yang, X.B. Ren, Phase diagram of polar states in doped ferroelectric systems, Physical Review B, 86 (2012).

[43] N. Zhou, C. Shen, M.F.X. Wagner, G. Eggeler, M.J. Mills, Y. Wang, Effect of $\mathrm{Ni}_{4} \mathrm{Ti}_{3}$ precipitation on martensitic transformation in Ti-Ni, Acta Materialia, 58 (2010) 6685-6694.

[44] R. Groger, T. Lookman, A. Saxena, Defect-induced incompatibility of elastic strains: Dislocations within the Landau theory of martensitic phase transformations, Physical Review B, 78 (2008) 184101. 


\section{Figureand Table Captions}

Figure 1Schematic figure for the model system (NiTi alloys) with doping, left figure show the defect-free B2 unit cell, right figure showsBCC unit cell caused by excess Nidoped inB2 NiTi system.

Figure2 (a)-(f) calculated volume fraction of Rmartensite phase/domains for different defect strengthcoefficient $\phi$ indefect concentration $\mathrm{c}=0.03$ upon cooling (black) and heating (red). (g) The temperature hysteresis $(\Delta \mathrm{T})$ upon cooling and heating with different defect strength $\phi . \phi_{\mathrm{c}}$ is the critical defect strength coefficient value resulting in an almost zero hysteresis. The temperature hysteresis is calculated through measuring the temperature difference between cooling and heating when the volume fraction of martensite is $50 \%$.

Figure 3 The critical defect strength coefficient $\phi_{c}$ of R strain glass transition for different defect concentration according to temperature hysteresis simulations.

Figure 4Calculated phase diagram of $\mathrm{R}$ martensitic phase transition/strain glass transition upon cooling with different defect strength $\phi i n c=0.03 . \mathrm{T}_{\mathrm{S}}$ describes the phase transition start temperature where the volume fraction of martensite is $5 \%, \mathrm{~T}_{\mathrm{F}}$ describes the phase transition finish temperature where the volume fraction of martensite is $90 \%$. Insets describe the related microstructure, red, blue, yellow and green colors describe the four variants of $\mathrm{R}$ martensitic phase respectively.

Figure5 (a)-(f) calculated volume fraction of B19 martensite phase/domains for 
different defect strengthcoefficient $\phi a t \mathrm{c}=0.03$ upon cooling and heating (arrows). (g) The temperature hysteresis $(\Delta \mathrm{T})$ upon cooling and heating with different defect strength coefficient $\phi . \phi_{\mathrm{c}}$ is the critical coefficient value resulting in an almost zero hysteresis. (h) The microstructure of B19 phase at low temperature with different defect strength coefficient,different colors describe different martensitic variants respectively.

Figure 6The ratio between calculated maximum interaction energy of single defect-martensitic variants $\left(E_{M-D}^{\max }\right)$ and maximum interaction energy of different martensitic variants $\left(E_{M-M}^{\max }\right)$ changes as a function of defect strength coefficient $\phi$ at $\mathrm{c}=0.03 . \quad \phi_{C}^{R}$ and $\phi_{C}^{B 19}$ describe the critical defect strength coefficient for R strain glass and B19 strain glass according to Fig. 2 and Fig. 5.

Figure 7Stress field of (a) $\sigma_{11}$, (b) $\sigma_{22}$, (c) $\sigma_{33}$, (d) $\sigma_{12}$, (e) $\sigma_{13}$, (f) $\sigma_{23}$ caused by a single defect ( $\phi=8.76)$ in the center of the system (black color), purple and light blue colors represent the positive and negative values of the stress value respectively. (g) Negative excess interaction energy (low martensitic phase nucleation barrier) between different $\mathrm{R}$ martensitic variants and the defect;red, blue, green and yellow colors represent energy favored $4 \mathrm{R}$ martensitic variantsrespectively.

Figure 8Calculated stress field distribution probability of $(a) \sigma_{11}$ and $(b) \sigma_{12}$ inc $=0.03$ with different defect strength (from $\phi=1.75$ to $\phi=8.76$ ). (c) The distribution probability of interaction energy between local stress field caused by point defects and martensitic variant 1 with different defect strength (from $\phi=1.75$ to $\phi=8.76$ ). 
Figure 9(a) Spatial distribution of simulation grids containing point defects (white color). (b)-(f) Spatial distribution of local Von Mises stress $\left(\sigma_{\mathrm{VM}}\right)$ caused by point defects with different defect strength coefficient $\phi$.

Figure 10The calculation of average free energy density change of the whole system upon cooling with different defect strength(from $\phi=0.0$ to $\phi=8.76$ ) at $\mathrm{c}=0.03$. (a) Local chemical free energy, $F_{c h} .(b)$ The non-local gradient energy, $F_{g r}$. (c)The excess energy caused by defects, $F_{L}$.(d) The coherency elastic strain energy, $F_{\text {el }}$ (e) Total free energy, $F_{\text {total, }}$ and (f) Free energy without defects' effect, $F_{\text {total }}-F_{L}$.

Figure 11Average free energy density evolution of the whole systemwith timeafter removing defects' effect (i.e.,removing excess energy $F_{L}$ caused by defects) at $c=0.03$. Insets describe the related microstructure evolution. Black dot (black arrow) describes the initial free energy of nanosized martensitic domains got from $\mathrm{T}=0.8 \mathrm{~T}_{0}$ and $\phi=7.0$, blue circles describe the free energy evolution after removing excess energy $F_{L}$.

Table 1 The dimensionless parameters (with superscript*) used in our simulations and the relationship between physical and dimensionless parameters. 
Figure 1

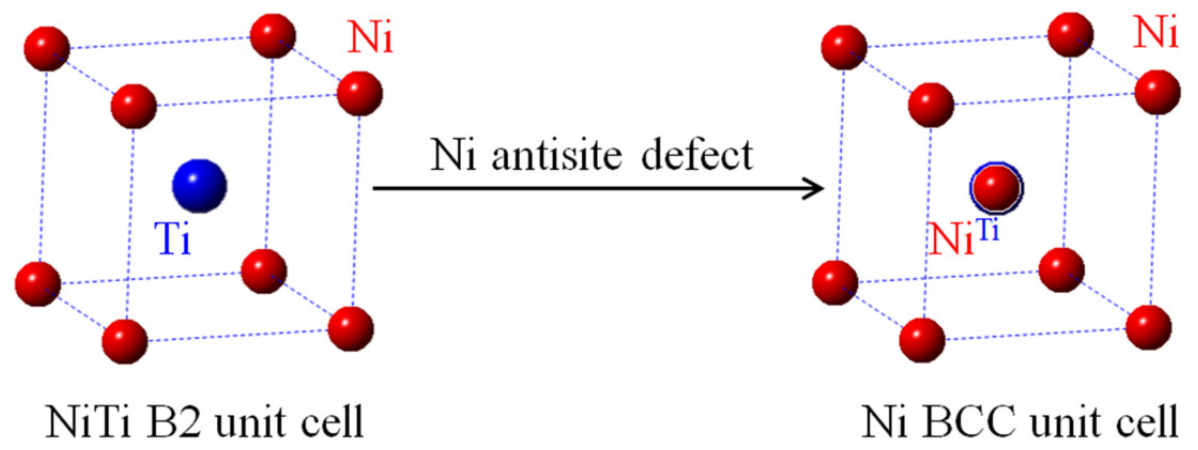


Figure 2

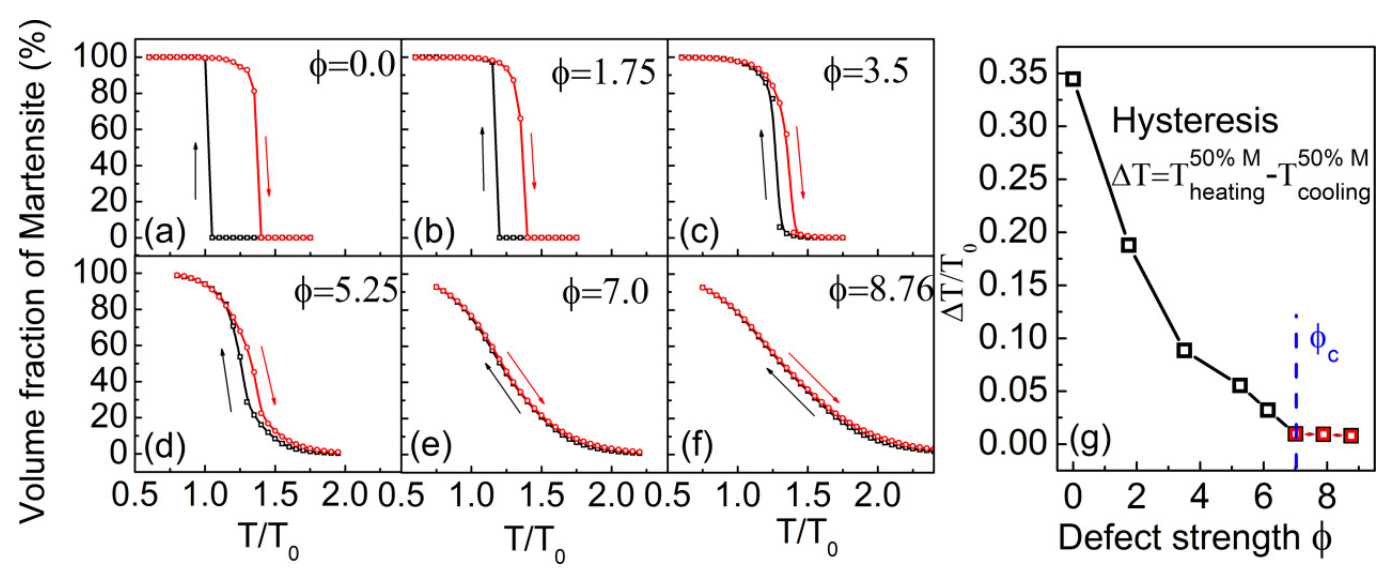


Figure 3

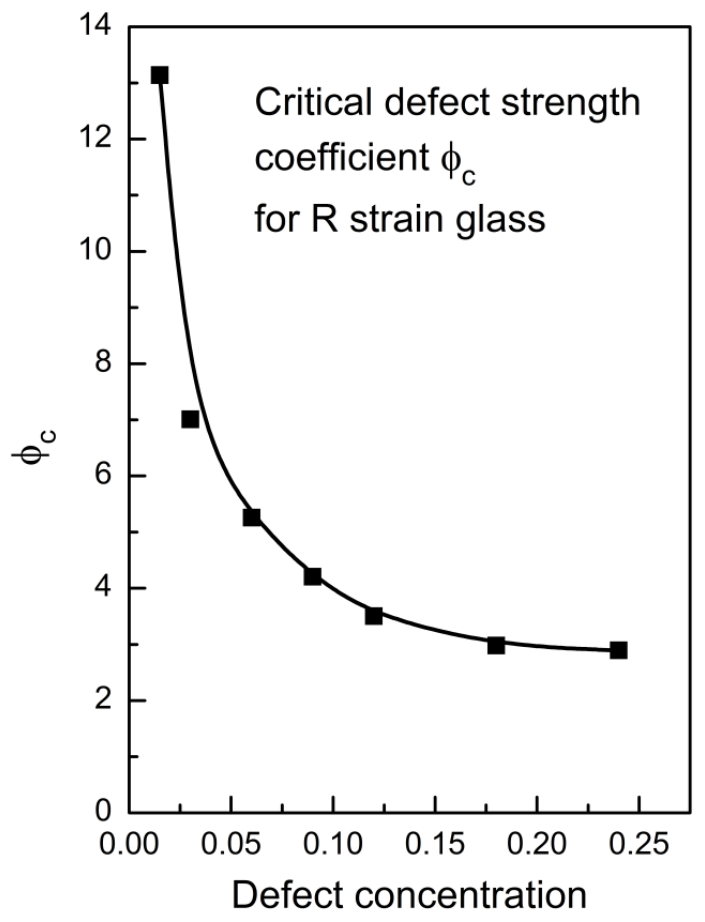


Figure 4

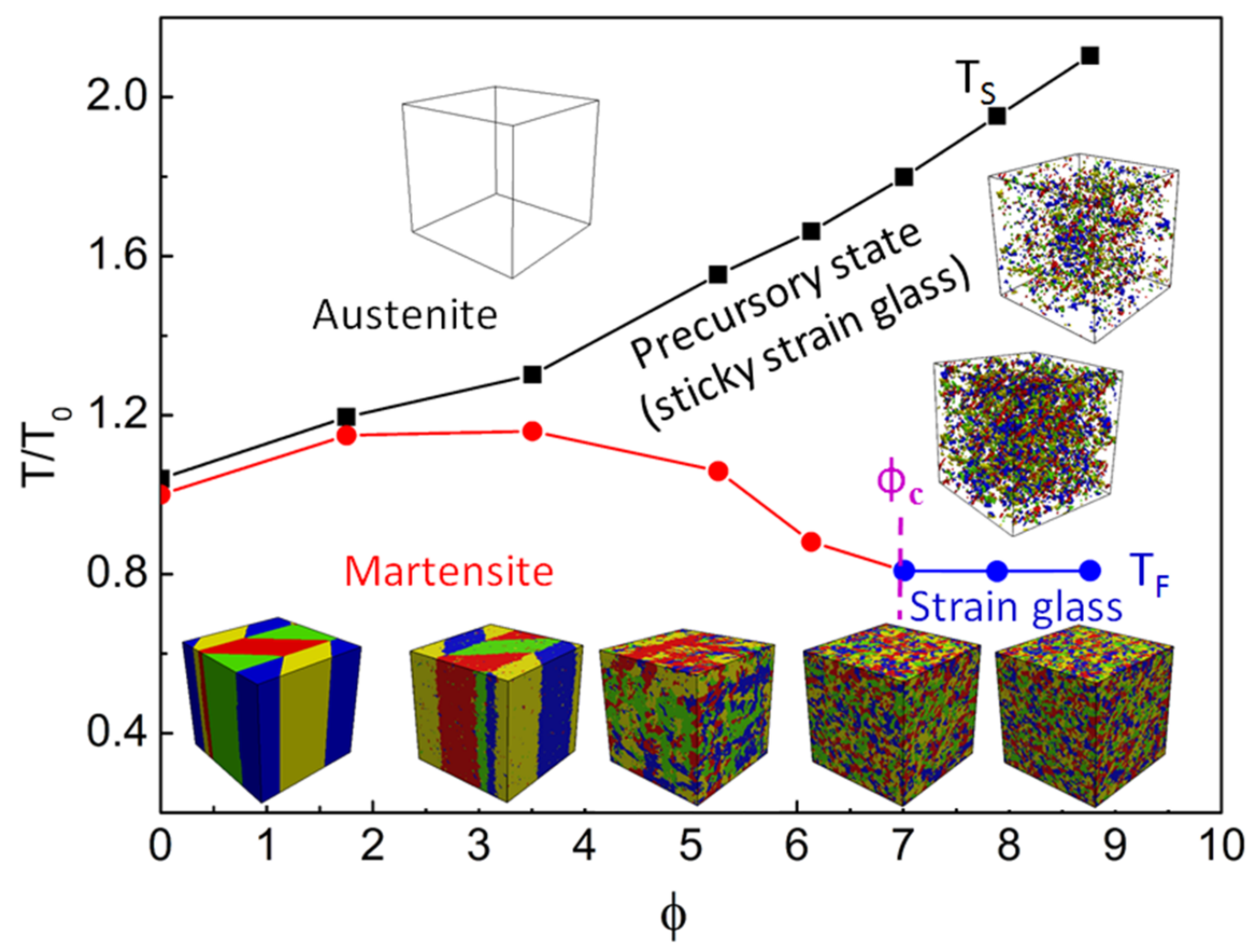


Figure 5

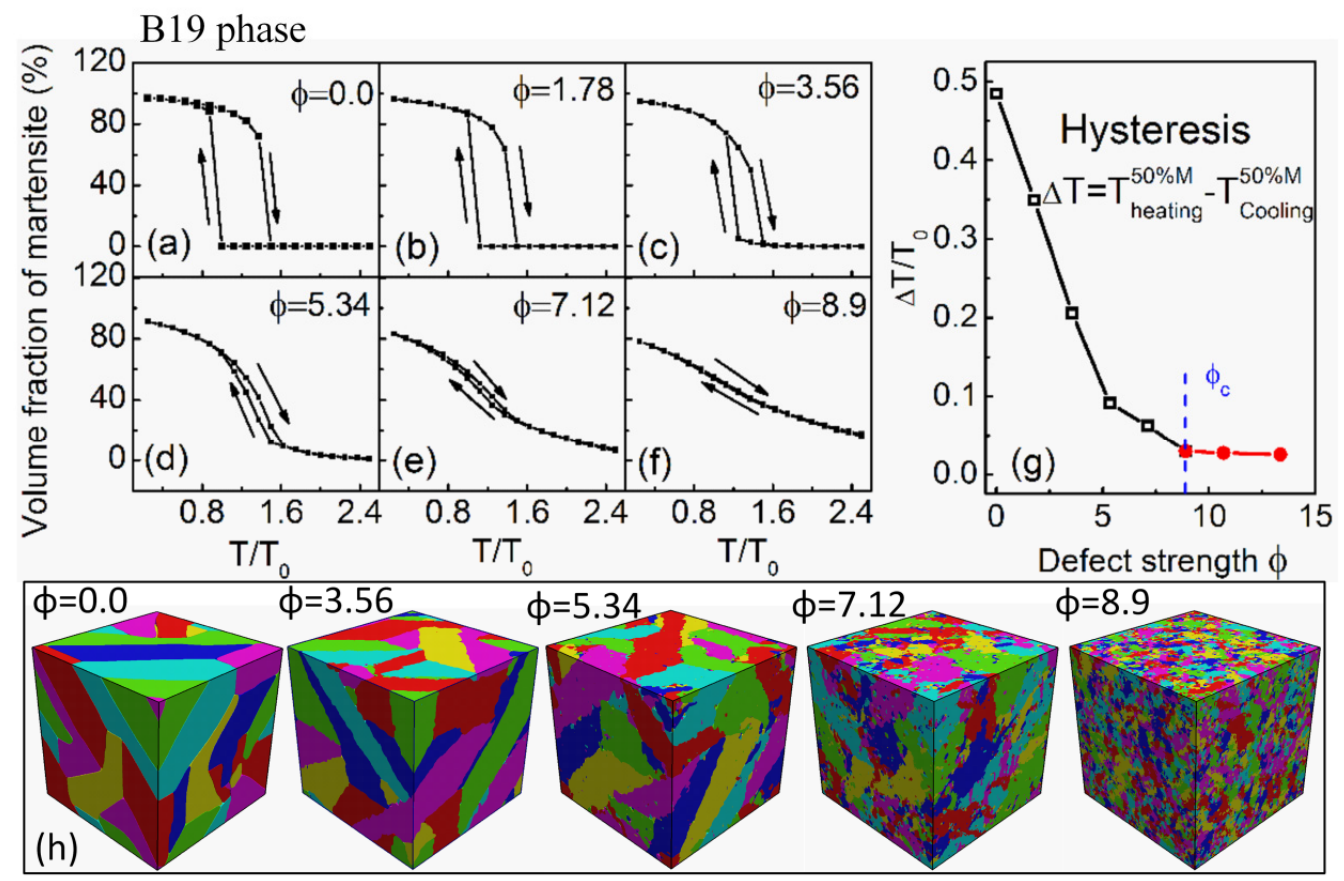


Figure 6

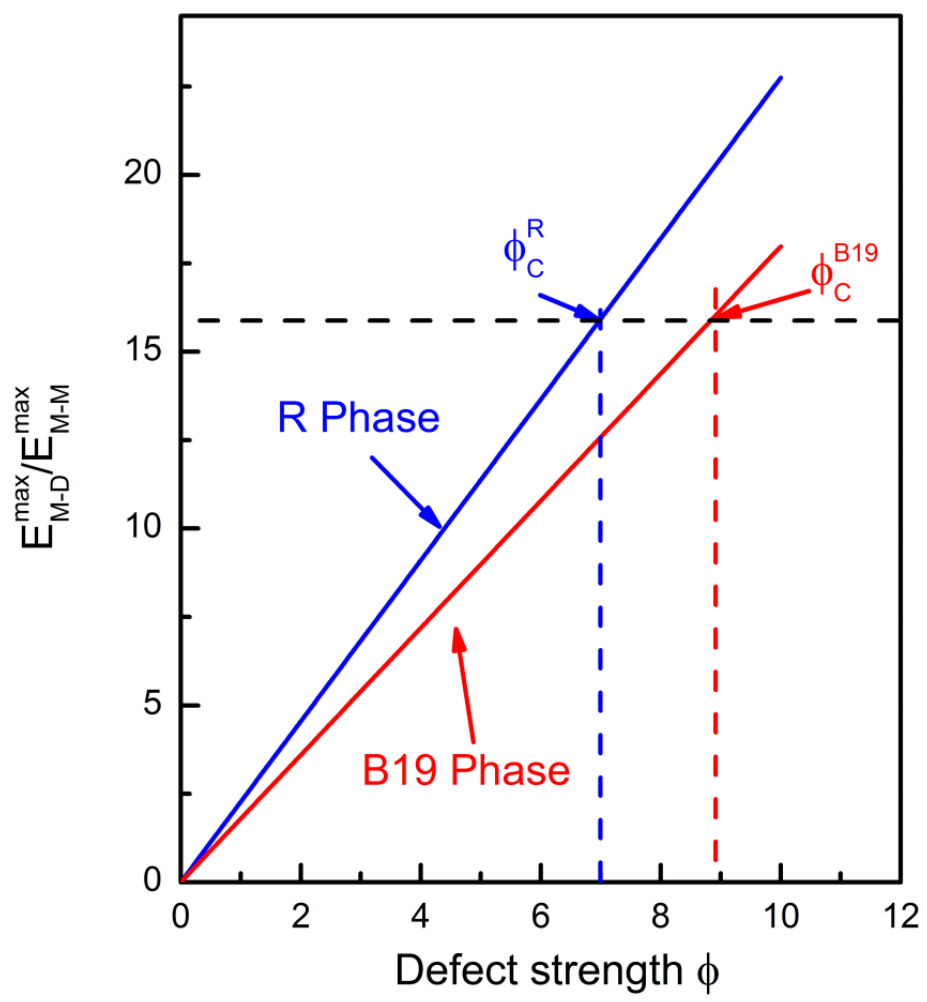


Figure 7
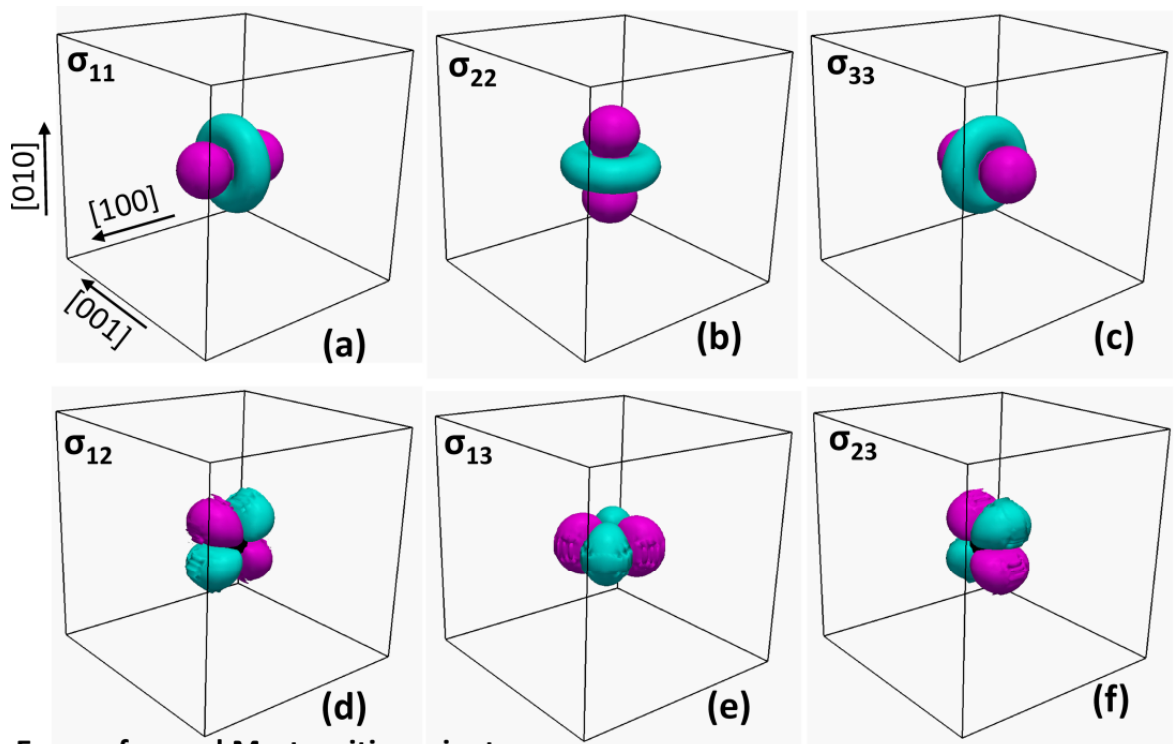

Energy favored Martensitic variants

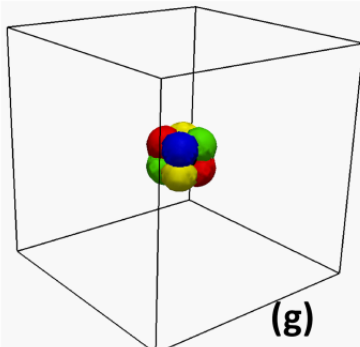

Defect position

Positive value of local stress

Negative value of local stress

Energy favored martensitic variant 1

Energy favored martensitic variant 2

Energy favored martensitic variant 3

Energy favored martensitic variant 4 
Figure 8
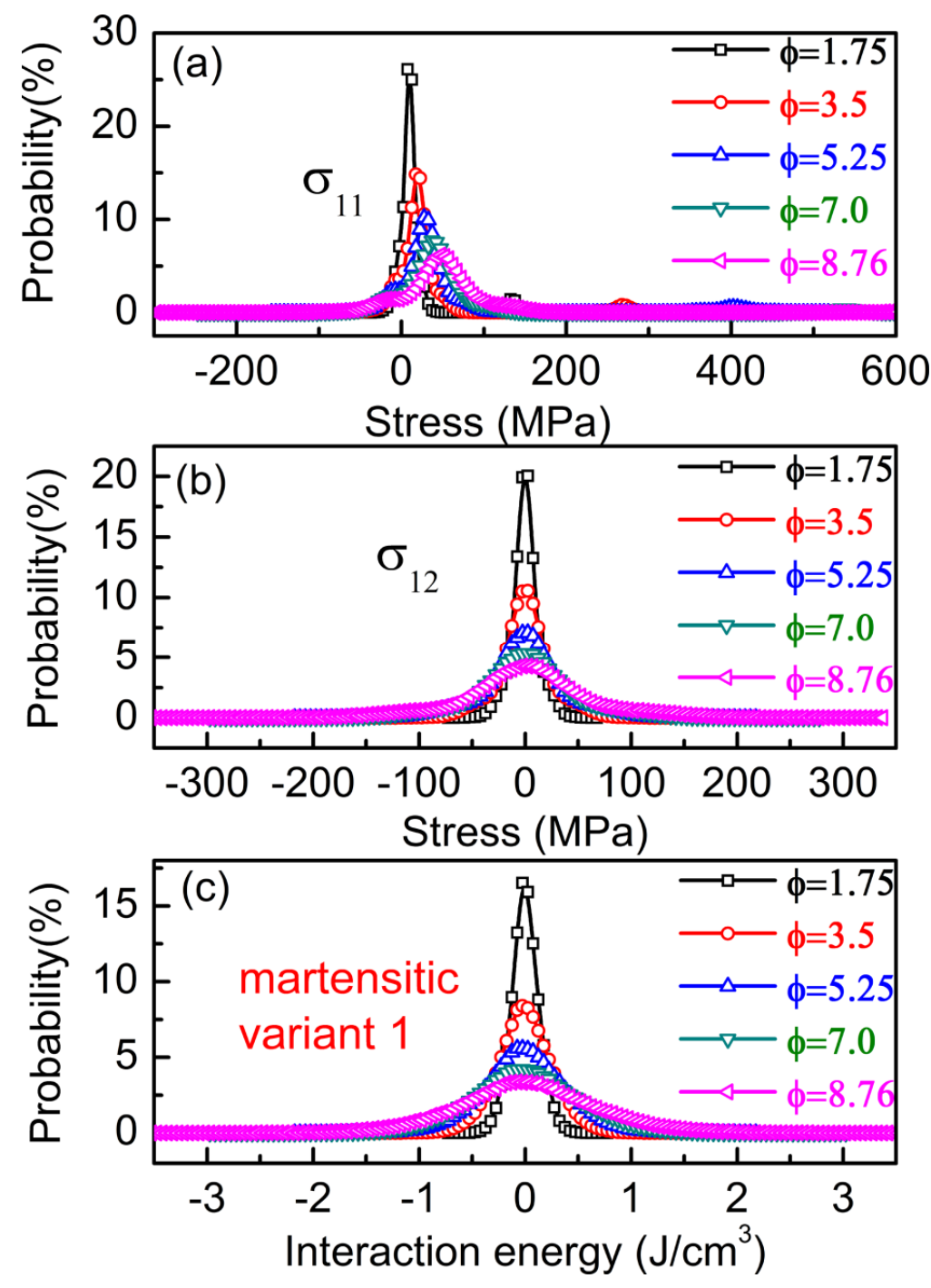
Figure 9

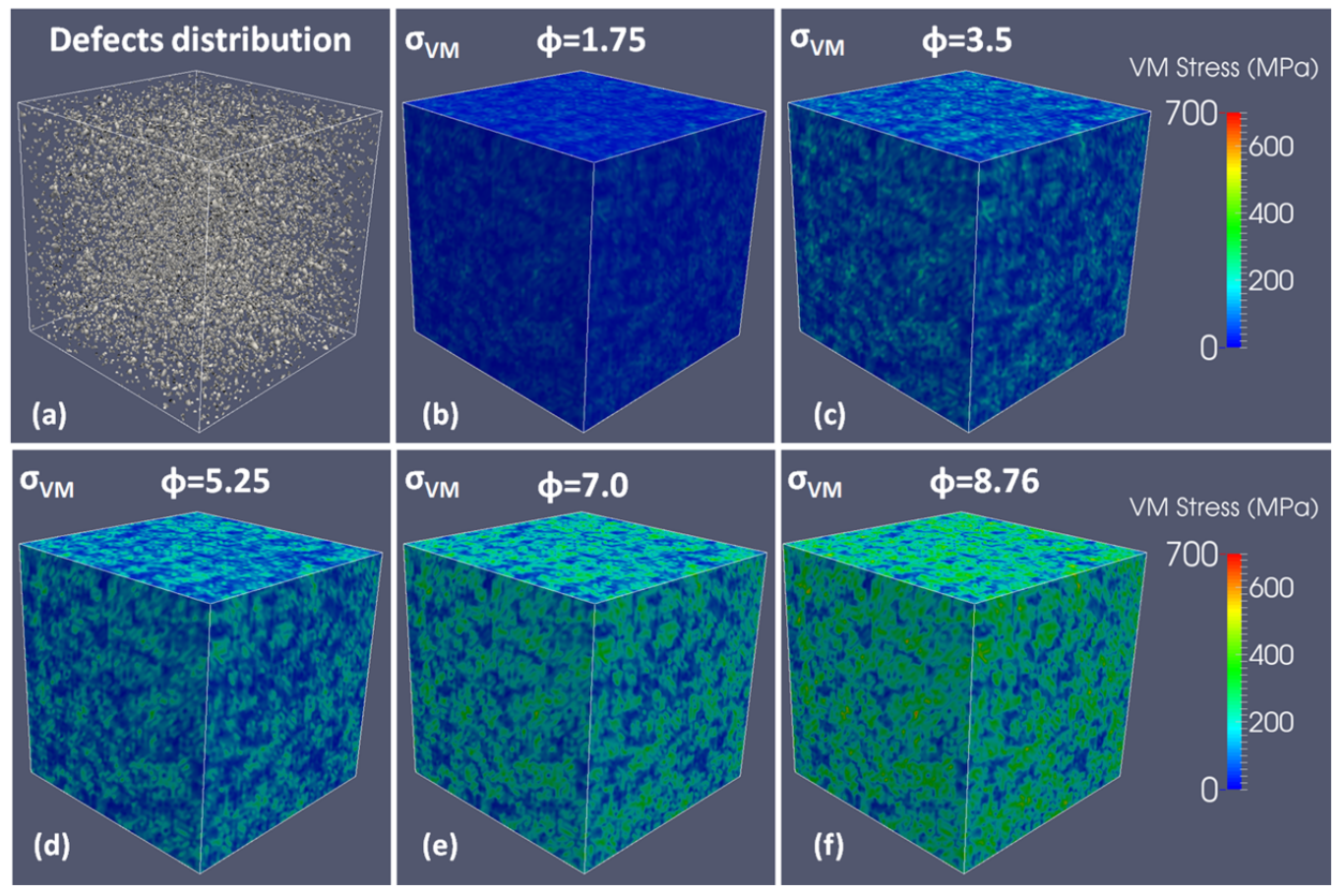


Figure 10
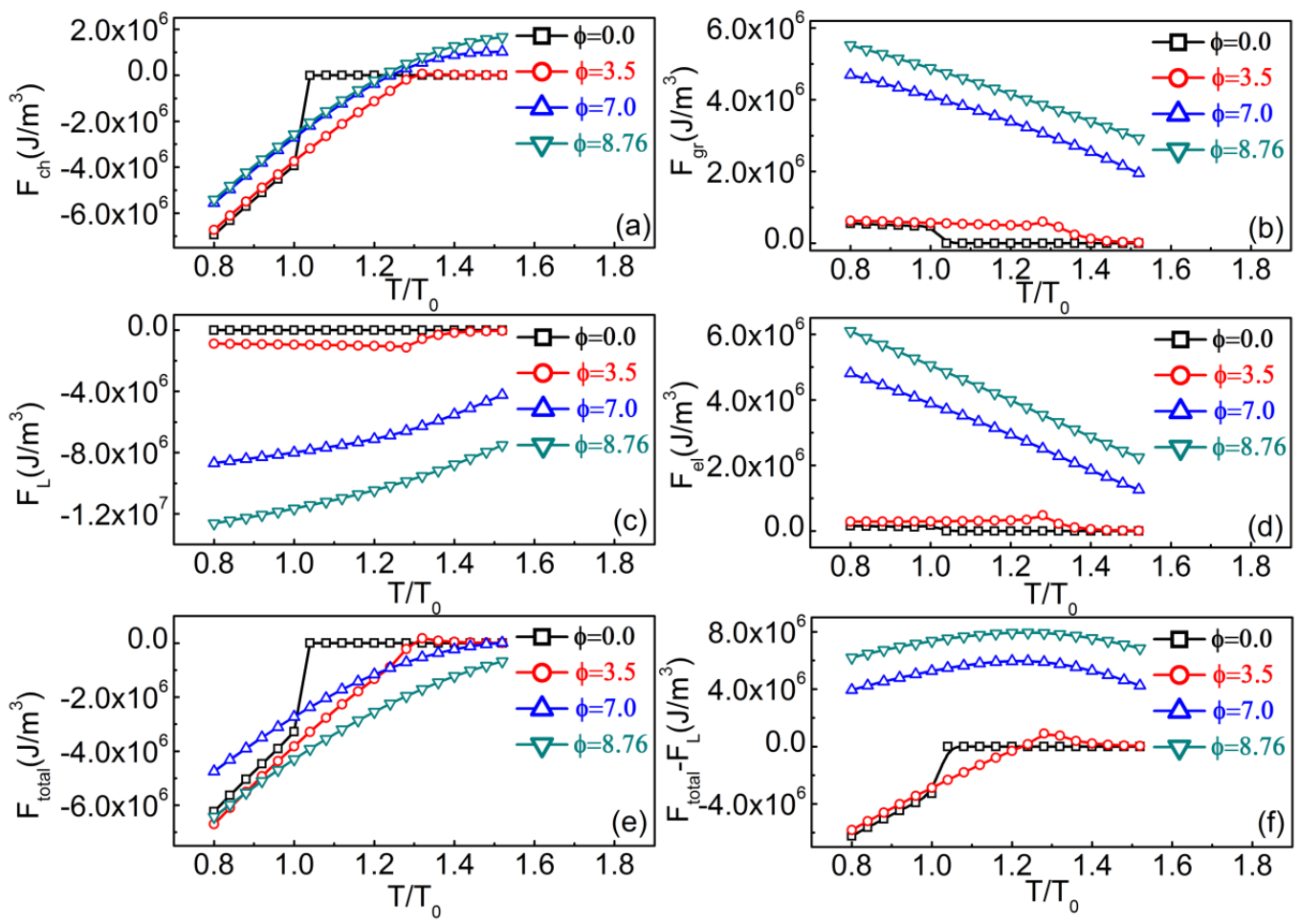
.Figure 11

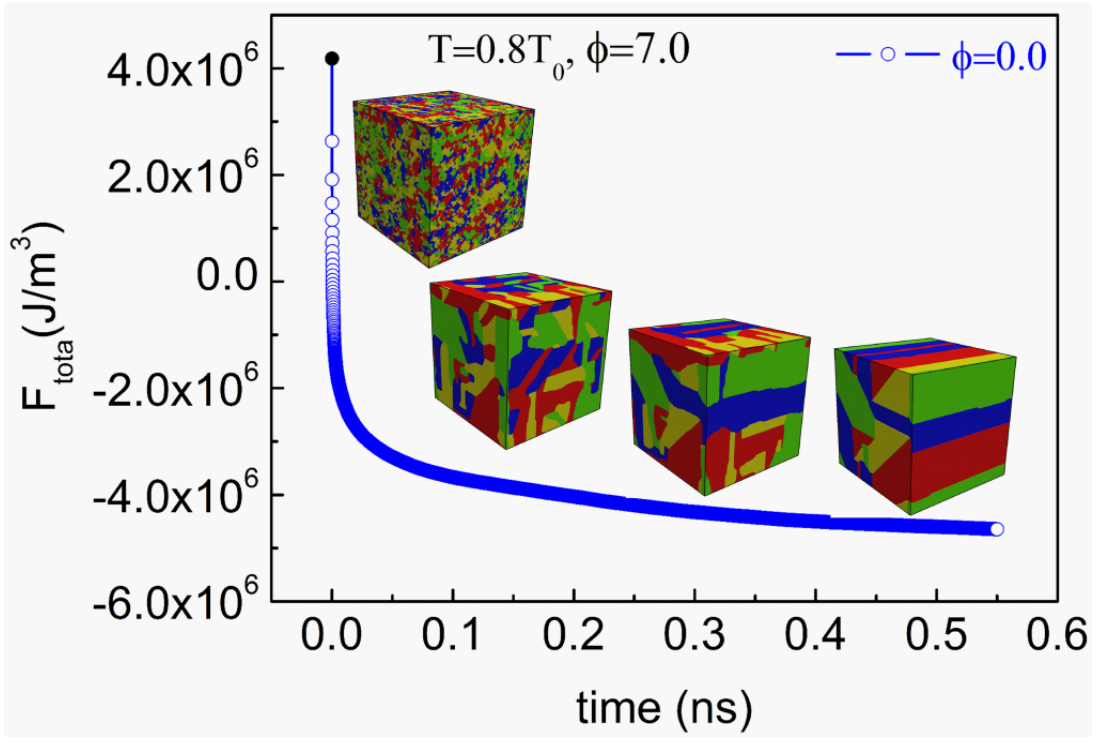


Table 1

\begin{tabular}{|c|c|c|c|c|c|}
\hline Elastic constants & Energy density & length & Fluctuation & Time & Dimensionless parameters \\
\hline$C_{i j}^{*}=C_{i j} / \theta$ & $\begin{array}{c}f_{c h}^{*}=f_{c h} / \theta \\
f_{L}^{*}=f_{L} / \theta \\
f_{g r}^{*}=f_{g r} /\left(\theta l_{o}\right)\end{array}$ & $\boldsymbol{r}^{*}=\boldsymbol{r} / l_{o}$ & $\xi_{p}^{*}=\xi_{p} /(M \theta)$ & $t^{*}=(t M \theta) /\left(M^{*}\right)$ & $\begin{array}{c}C_{11}^{*}=2 \times 10^{4}, C_{12}^{*}=1.5 \times 10^{4}, \\
C_{44}^{*}=5 \times 10^{3}, A_{1}^{0 *}=20, A_{2}^{*}=32.05, \\
A_{1}^{0 *}=37.5, \\
\beta^{*}=1.5, M^{*}=1, t^{*}=1\end{array}$ \\
\hline
\end{tabular}

An asterisk denotes a dimensionless quantity, $\theta=10^{7} \mathrm{~J} / \mathrm{m}^{3}$ is the energy scaling factor, $l_{\mathrm{o}}=1 \mathrm{~nm}$ is the grid size. 